\title{
Some Gradient Estimates on Covering Manifolds
}

\author{
by \\ Nick DUNGEY \\ Presented by Andrzej HULANICKI
}

\begin{abstract}
Summary. Let $M$ be a complete Riemannian manifold which is a Galois covering, that is, $M$ is periodic under the action of a discrete group $G$ of isometries. Assuming that $G$ has polynomial volume growth, we provide a new proof of Gaussian upper bounds for the gradient of the heat kernel of the Laplace operator on $M$. Our method also yields a control on the gradient in case $G$ does not have polynomial growth.
\end{abstract}

1. Introduction. Consider a complete, non-compact, connected Riemannian manifold $M$. Suppose that a finitely generated discrete group $G$ acts properly and freely on $M$ by isometries, such that the orbit space $M_{1}=M / G$ is a compact manifold. In other words, $M$ is a Galois covering manifold of the compact Riemannian manifold $M_{1}$, with deck transformation group (isomorphic to) $G$. In this paper, we study regularity properties of the heat kernel on $M$.

We will assume that $G$ has polynomial volume growth of some order $D \geq 1$. That is, after fixing a finite set $S \subseteq G$ of generators which is symmetric $\left(S=S^{-1}\right)$, one has an estimate $c^{-1} k^{D} \leq d g\left(S^{k}\right) \leq c k^{D}$ for all $k \in \mathbb{N}$, where $d g$ is the counting measure on $G$ and $S^{k}:=\left\{g_{1} \cdots g_{k}: g_{j} \in S\right\}$ (for background, see [9, Chapters VI and X]). Remark that the simplest case of our setting occurs with $M=\mathbb{R}^{D}$ endowed with a Riemannian metric which is periodic under the standard action of $G=\mathbb{Z}^{D}$ by translations.

Denote by $K_{t}(x, y), t>0, x, y \in M$, the heat kernel of the Laplace operator $H$ on $M$. Under our assumptions on $M$ and $G$, it is well known that one has, for some $c, b>0$, the Gaussian estimate

$$
K_{t}(x, y) \leq c V\left(x, t^{1 / 2}\right)^{-1} e^{-b d(x, y)^{2} / t}
$$

2000 Mathematics Subject Classification: 58J35, 35B40, 35B27.

Key words and phrases: heat kernel, Riemannian manifold, covering manifold, gradient estimates, periodic operator, polynomial growth. 
for all $t>0$ and $x, y \in M$ (see for example [7,8]). Here, $d(x, y)$ is the Riemannian distance and $V(x, r):=d x(B(x, r))$ is the Riemannian measure of the ball $B(x, r)=\{y \in M: d(x, y)<r\}$.

The following theorem was proved in [4]. Adopt the convention that $\nabla K_{t}(x, y)=\nabla_{x} K_{t}(x, y)$ denotes the gradient with respect to the first variable of the two-variable kernel $K_{t}(\cdot, \cdot)$.

TheOREM 1.1. There exist $c, b>0$ such that

$$
\left|\nabla K_{t}(x, y)\right| \leq c t^{-1 / 2} V\left(x, t^{1 / 2}\right)^{-1} e^{-b d(x, y)^{2} / t}
$$

for all $t>0$ and $x, y \in M$.

In this paper, we give an alternative proof of Theorem 1.1 which is more direct than that of [4], and does not depend on a global parabolic Harnack inequality or Hölder regularity estimates from [8]. Instead, it depends on (1) and its standard consequences, together with the periodicity (i.e., $G$ invariance) of the Laplace operator $H$.

We remark that our proof could be adapted to give a new proof of gradient estimates for second-order, divergence-form elliptic operators on $\mathbb{R}^{D}$ with smooth, possibly complex, periodic coefficients (see [5] and references therein).

Moreover, our method gives a certain control over the gradient on general covering manifolds, without any assumption of polynomial growth. See Remark 2.4 below for a new inequality in this situation.

As an interesting application of Theorem 1.1, note that recent work [1] allows one to deduce from Theorem 1.1 that the Riesz transform $\nabla H^{-1 / 2}$ is bounded in $L^{p}(M)$ for all $1<p<\infty$. The boundedness of the Riesz transform was obtained by different methods in [4].

2. Proof of Theorem 1.1. In general, $c, c^{\prime}, b$ and so on denote positive constants whose value may change from line to line when convenient. One has the standard volume estimates

$$
\begin{array}{ll}
c^{-1} r^{n} \leq V(x, r) \leq c r^{n}, & 0<r<1, \\
c^{-1} r^{D} \leq V(x, r) \leq c r^{D}, & r \geq 1,
\end{array}
$$

uniformly for all $x \in M$, where $n$ is the local Euclidean dimension of $M$ and $D$ is the order of polynomial growth of $G$. Denote the action of $G$ on $M$ by $g \cdot x=g x$ for $g \in G, x \in M$. In what follows, we fix a relatively compact, open fundamental domain $X \subseteq M$ : thus the sets $g X:=\{g x: x \in X\}$, $g \in G$, are pairwise disjoint subsets of $M$, and $M \backslash\left(\bigcup_{g \in G} g X\right)$ is a set of measure zero.

Using a local Harnack inequality for solutions of the heat equation (for example, [9, Theorem V.5.1]), one may deduce from (1) the estimate of 
Theorem 1.1 for small times: for any $t_{0} \in(0, \infty)$, one has an estimate

$$
\left|\nabla K_{t}(x, y)\right| \leq c t^{-1 / 2} t^{-n / 2} e^{-b d(x, y)^{2} / t}
$$

for all $t \in\left(0, t_{0}\right]$ and $x, y \in M$ (for details see [4, Theorem 2.4]). Thus, to get Theorem 1.1 it remains to show that, for some $t_{0}>0$, one has

$$
\left|\nabla K_{t}(x, y)\right| \leq c t^{-1 / 2} t^{-D / 2} e^{-b d(x, y)^{2} / t}
$$

for all $t \geq t_{0}$ and $x, y \in M$.

By general methods (see for example [3, 6]), one obtains from (1) estimates of the time derivatives of $K_{t}$. Thus there is a $b>0$ such that, for any $k \in \mathbb{N}_{0}=\{0,1,2, \ldots\}$, there exists $c>0$ with

$$
\left|\partial_{0}^{k} K_{t}(x, y)\right| \leq c t^{-k} V\left(x, t^{1 / 2}\right)^{-1} e^{-b d(x, y)^{2} / t}
$$

for all $t>0$ and $x, y \in M$, where $\partial_{0}=\partial / \partial t$ denotes the time derivative. An integration of estimates (3) shows that there exists $\alpha>0$ such that, given any $k \in \mathbb{N}_{0}$, one has an estimate of form

$$
\int_{M} d y e^{\alpha d(x, y)^{2} / t}\left|\partial_{0}^{k} K_{t}(x, y)\right|^{2} \leq c t^{-2 k} V\left(x, t^{1 / 2}\right)^{-1} \leq c^{\prime} t^{-2 k} t^{-D / 2}
$$

for all $t \geq 1$ and $x \in M$. Using (3), one may also argue (see [6] or [2]) that there is $\alpha>0$ such that

$$
\int_{M} d x e^{\alpha d(x, y)^{2} / t}\left|\nabla K_{t}(x, y)\right|^{2} \leq c t^{-1} V\left(y, t^{1 / 2}\right)^{-1}
$$

for all $y \in M$ and $t>0$. Integrating this estimate over $y \in X$ yields

$$
\int_{M} d x \int_{X} d y e^{\alpha d(x, y)^{2} / t}\left|\nabla K_{t}(x, y)\right|^{2} \leq c t^{-1} t^{-D / 2}
$$

for all $t \geq 1$. Now observe:

LEMMA 2.1. Let $P: M \times M \rightarrow[0, \infty)$ be a measurable function which is $G$-invariant, that is, $P(g x, g y)=P(x, y)$ for all $g \in G$ and $x, y \in M$. Then

$$
\int_{M} d x \int_{X} d y P(x, y)=\int_{X} d x \int_{M} d y P(x, y)
$$

Proof. The left side equals

$$
\begin{aligned}
\int_{G} d g \int_{X} d y \int_{X} d x P(g x, y) & =\int_{G} d g \int_{X} d y \int_{X} d x P\left(x, g^{-1} y\right) \\
& =\int_{X} d x \int_{M} d y P(x, y),
\end{aligned}
$$

as required. 
The kernel $(x, y) \mapsto e^{\alpha d(x, y)^{2} / t}\left|\nabla K_{t}(x, y)\right|^{2}$ is $G$-invariant, so we may rewrite (5) as

$$
\int_{X} d x \int_{M} d y e^{\alpha d(x, y)^{2} / t}\left|\nabla K_{t}(x, y)\right|^{2} \leq c t^{-1} t^{-D / 2}
$$

for all $t \geq 1$. The following lemma is essentially a local regularity estimate for $K_{t}$; we postpone the proof. Denote by $e$ the identity of $G$.

Lemma 2.2. There exists a finite set $A \subseteq G$ with $e \in A$ such that, setting $A \cdot X=\{g x: g \in A, x \in X\} \subseteq M$, one has

$$
\left|\nabla K_{t}(u, y)\right|^{2} \leq c \int_{t-1}^{t+1} d s \int_{A \cdot X} d x\left(\left|\nabla K_{s}(x, y)\right|^{2}+\left|\partial_{0} K_{s}(x, y)\right|^{2}\right)
$$

for all $t \geq 2, u \in X$ and $y \in M$.

The triangle inequality gives $d(u, y)^{2} \leq 2\left(d(u, x)^{2}+d(x, y)^{2}\right) \leq 2 d(x, y)^{2}$ $+2 c_{0}^{2}$ for all $u \in X, x \in A \cdot X$ and $y \in M$, where $c_{0}=\sup \left\{d\left(x_{1}, x_{2}\right): x_{1}, x_{2}\right.$ $\in A \cdot X\}<\infty$. Therefore, by multiplying both sides of the estimate in Lemma 2.2 by $e^{\beta d(u, y)^{2} / t}$, for some constants $c, c^{\prime}>1$ one obtains

$$
\begin{aligned}
e^{\beta d(u, y)^{2} / t} \mid & \left.\nabla K_{t}(u, y)\right|^{2} \\
& \leq c \int_{t-1}^{t+1} d s \int_{A \cdot X} d x e^{c^{\prime} \beta d(x, y)^{2} / s}\left(\left|\nabla K_{s}(x, y)\right|^{2}+\left|\partial_{0} K_{s}(x, y)\right|^{2}\right)
\end{aligned}
$$

for all $\beta \geq 0, t \geq 2, u \in X$, and $y \in M$. Let $\alpha>0$ be such that estimates (4) and (6) hold, and set $\beta=\left(c^{\prime}\right)^{-1} \alpha$. Integrating the last estimate over $y \in M$ yields

$$
\int_{M} d y e^{\beta d(u, y)^{2} / t}\left|\nabla K_{t}(u, y)\right|^{2}
$$

$$
\leq c \int_{t-1}^{t+1} d s \int_{X} d x \int_{M} d y e^{\alpha d(x, y)^{2} / s}\left(\left|\nabla K_{s}(x, y)\right|^{2}+\left|\partial_{0} K_{s}(x, y)\right|^{2}\right) \leq c^{\prime} t^{-1} t^{-D / 2}
$$

for all $t \geq 2$ and $u \in X$. (Here, for the first inequality we used the fact that a $G$-invariant kernel $P$ satisfies $\int_{A \cdot X} d x \int_{M} d y P(x, y)=c_{1} \int_{X} d x \int_{M} d y P(x, y)$, where $c_{1}$ is the finite cardinality of $A$.) Then for all $u \in X$ and $z \in M$, by writing $d(u, z)^{2} \leq 2 d(u, y)^{2}+2 d(y, z)^{2}$, we deduce for some $\gamma>0$ that

$$
\begin{aligned}
& e^{\gamma d(u, z)^{2} / t}\left|\nabla K_{t}(u, z)\right| \\
& \quad \leq \int_{M} d y e^{2 \gamma d(u, y)^{2} / t}\left|\nabla K_{t / 2}(u, y)\right| e^{2 \gamma d(y, z)^{2} / t}\left|K_{t / 2}(y, z)\right| \\
& \quad \leq\left(\int_{M} d y e^{4 \gamma d(u, y)^{2} / t}\left|\nabla K_{t / 2}(u, y)\right|^{2}\right)^{1 / 2}\left(\int_{M} d y e^{4 \gamma d(z, y)^{2} / t}\left|K_{t / 2}(z, y)\right|^{2}\right)^{1 / 2} \\
& \quad \leq c t^{-1 / 2} t^{-D / 2}
\end{aligned}
$$


for all $t \geq 4$, where the second step used the symmetry $K_{s}(y, z)=K_{s}(z, y)$ and the last step used (7) and (4). Since $K_{t}$ is $G$-invariant, this establishes (2) for all $t \geq 4$ and $x, y \in M$, and Theorem 1.1 follows.

It remains to prove Lemma 2.2. We need the following, rather crude local estimate which is valid for an arbitrary Riemannian manifold $M$. By a harmonic function we mean a function $F$ which satisfies the heat equation $\left(\partial_{0}+H\right) F=0$ in some open set $V^{\prime} \subseteq \mathbb{R} \times M$.

LEMMA 2.3. Let $V^{\prime}$ be an open subset of $\mathbb{R} \times M$ and $K^{\prime}$ be a compact subset of $V^{\prime}$. Then there exists $c>0$ such that

$$
\left\|\partial_{0} F\right\|_{L^{\infty}\left(K^{\prime}\right)}+\|\nabla F\|_{L^{\infty}\left(K^{\prime}\right)} \leq c\left(\left\|\partial_{0} F\right\|_{L^{2}\left(V^{\prime}\right)}+\|\nabla F\|_{L^{2}\left(V^{\prime}\right)}\right)
$$

for all functions $F$ harmonic in $V^{\prime}$. (Here, the $L^{2}$ norm is taken with respect to the measure $d t d x$ on $\mathbb{R} \times M$.)

Proof. Since the desired estimate is local in nature, without loss of generality we may assume that $V^{\prime}$ is a small ball in the Riemannian manifold $\widetilde{M}=\mathbb{R} \times M$. Choose a ball $U^{\prime}$ with $K^{\prime} \subseteq U^{\prime} \subseteq \overline{U^{\prime}} \subseteq V^{\prime}$. Let $\widetilde{\nabla}$ denote the gradient for $\widetilde{M}$. Because the operator $\partial_{0}+H$ is hypoelliptic, one has an estimate

$$
\|\widetilde{\nabla} F\|_{L^{\infty}\left(K^{\prime}\right)} \leq c\|F\|_{L^{2}\left(U^{\prime}\right)}
$$

for all functions $F$ harmonic in $U^{\prime}$ (see for example [9, Corollary III.1.3]). Let $F$ be a harmonic function in $V^{\prime}$ and set $a=\left(\operatorname{vol} U^{\prime}\right)^{-1} \int_{U^{\prime}} F$, the average of $F$ on $U^{\prime}$. From (8) and a local Poincaré inequality for balls of $\widetilde{M}$, we obtain

$$
\|\widetilde{\nabla} F\|_{L^{\infty}\left(K^{\prime}\right)}=\|\widetilde{\nabla}(F-a)\|_{L^{\infty}\left(K^{\prime}\right)} \leq c\|F-a\|_{L^{2}\left(U^{\prime}\right)} \leq c^{\prime}\|\widetilde{\nabla} F\|_{L^{2}\left(V^{\prime}\right)} .
$$

This proves the lemma.

To prove Lemma 2.2, choose a relatively compact, open set $U \subseteq M$ with $\bar{X} \subseteq U$. There exists a finite set $A \subseteq G$, with $e \in A$, such that the set $U \backslash(A \cdot X)$ has measure zero. We can apply Lemma 2.3 with $V^{\prime}=(-1,1) \times U$ and $K^{\prime}=[-1 / 2,1 / 2] \times \bar{X}$ to the harmonic functions $F_{(t, y)}, y \in M, t \geq 2$, defined by $F_{(t, y)}(s, x):=K_{t+s}(x, y)$ for $s>-t, x \in M$. Then Lemma 2.2 follows easily. The proof of Theorem 1.1 is complete.

REMARK 2.4. Let us explain some general inequalities which relate to the above proof. Adapting notation of [6], we consider the quantities

$$
\begin{array}{ll}
E_{0}(y, t)=\int_{M} d x K_{t}(x, y)^{2}=K_{2 t}(y, y), & E_{2}(y, t)=\int_{M} d x\left|\partial_{0} K_{t}(x, y)\right|^{2}, \\
E_{1}(y, t)=\int_{M} d x\left|\nabla K_{t}(x, y)\right|^{2}, & \widetilde{E}_{1}(y, t)=\int_{M} d x\left|\nabla K_{t}(y, x)\right|^{2}
\end{array}
$$

for $t>0$ and $y \in M$. Grigor'yan [6] shows on arbitrary manifolds that any estimate of the form $E_{0}(y, t) \leq 1 / f(t), t>0$, leads to upper estimates of 
$E_{1}(y, \cdot)$ and $E_{2}(y, \cdot)$. In fact, one has general inequalities $([6$, p. 372])

$$
E_{i}(y, t) \leq c\left(\int_{0}^{t} d \tau\left(E_{i-1}(y, \tau)\right)^{-1}\right)^{-1}
$$

for $i=1,2$ and all $t>0$. On the other hand, the gradient of the heat kernel is estimated in terms of $\widetilde{E}_{1}$ and $E_{0}$ by

$$
\left|\nabla K_{2 t}(x, y)\right| \leq\left(\widetilde{E}_{1}(x, t)\right)^{1 / 2}\left(E_{0}(y, t)\right)^{1 / 2}
$$

for any $x, y \in M$. We claim that

$$
\sup _{u \in M} \widetilde{E}_{1}(u, t) \leq c \int_{X} d x E_{1}(x, t-1)
$$

for all $t \geq 2$, where $X$ is a fundamental domain. Inequality (10) is valid for any Galois covering manifold $M$, that is, no assumption of polynomial growth is required. Thus on covering manifolds, the above remarks allow one to estimate $\left|\nabla K_{t}(x, y)\right|$ given knowledge of the quantity $E_{0}$. For estimates of $E_{0}$ on covering manifolds, see for example [7].

The proof of (10) is a variation of the proof of Theorem 1.1. First observe that, by $G$-invariance, $\sup _{u \in M} \widetilde{E}_{1}(u, t)=\sup _{u \in X} \widetilde{E}_{1}(u, t)$. Then integrate the estimate of Lemma 2.2 over $y \in M$ (with a time interval of length 1 instead of 2), and apply Lemma 2.1 to obtain

$$
\sup _{u \in X} \widetilde{E}_{1}(u, t) \leq c \int_{t-(1 / 2)}^{t+(1 / 2)} d s \int_{X} d x\left(E_{1}(x, s)+E_{2}(x, s)\right)
$$

for all $t \geq 2$. The functions $t \mapsto E_{i}(x, t)$ are non-increasing: see [6]. By taking an interval of integration $[t-1 / 2, t]$ in $(9)$, one easily sees that $E_{2}(x, t) \leq$ $c^{\prime} E_{1}(x, t-1 / 2)$ for all $t>1 / 2$. Then (10) follows.

Acknowledgements. This work was carried out with financial support from the Australian Research Council (ARC) Centre of Excellence for Mathematics and Statistics of Complex Systems (MASCOS).

\section{References}

[1] P. Auscher, T. Coulhon, X. T. Duong and S. Hofmann, Riesz transform on manifolds and heat kernel regularity, preprint.

[2] T. Coulhon and X. T. Duong, Riesz transforms for $1 \leq p \leq 2$, Trans. Amer. Math. Soc. 351 (1999), 1151-1169.

[3] E. B. Davies, Non-Gaussian aspects of heat kernel behaviour, J. London Math. Soc. 55 (1997), 105-125.

[4] N. Dungey, Heat kernel estimates and Riesz transforms on some Riemannian covering manifolds, Math. Z. 247 (2004), 765-794.

[5] A. F. M. ter Elst, D. W. Robinson and A. Sikora, On second-order periodic elliptic operators in divergence form, Math. Z. 238 (2001), 569-637. 
[6] A. Grigor'yan, Upper bounds of derivatives of the heat kernel on an arbitrary complete manifold, J. Funct. Anal. 127 (1995), 363-389.

[7] - Estimates of heat kernels on Riemannian manifolds, in: Spectral Theory and Geometry, B. Davies and Y. Safarov (eds.), London Math. Soc. Lecture Note Ser. 273, Cambridge Univ. Press, Cambridge, 1999, 140-225.

[8] L. Saloff-Coste, A note on Poincaré, Sobolev, and Harnack inequalities, Internat. Math. Res. Notices 1992, no. 2, 27-38.

[9] N. T. Varopoulos, L. Saloff-Coste and T. Coulhon, Analysis and Geometry on Groups, Cambridge Tracts in Math. 100, Cambridge Univ. Press, Cambridge, 1992.

Nick Dungey

School of Mathematics

The University of New South Wales

Sydney 2052, Australia

E-mail: dungey@maths.unsw.edu.au

Received August 31, 2004 